\title{
Preoperative Vision, Gender, and Operation Time Predict Visual Improvement After Epiretinal Membrane Vitrectomy: A Retrospective Study
}

\author{
Henry Bair ${ }^{1,2}$ \\ Wei-Hsun Kung (D) \\ Chun-Ting Lai ${ }^{1, *}$ \\ Chun-Ju Lin (iD) 1,3,4,* \\ Huan-Sheng Chen (iD ${ }^{5}$ \\ Cheng-Hsien Chang ${ }^{\prime}$ \\ Jane-Ming Lin' \\ Ning-Yi Hsia' \\ Wen-Lu Chen' \\ Peng-Tai Tien ${ }^{1,6}$ \\ Wen-Chuan Wu' \\ Yi-Yu Tsai ${ }^{1,3,4}$ \\ 'Department of Ophthalmology, China \\ Medical University Hospital, China \\ Medical University, Taichung, Taiwan; \\ ${ }^{2}$ Stanford University School of Medicine, \\ Stanford, CA, USA; ${ }^{3}$ School of Medicine, \\ College of Medicine, China Medical \\ University, Taichung, Taiwan; \\ ${ }^{4}$ Department of Optometry, Asia \\ University, Taichung, Taiwan; ${ }^{5}$ An-Shin \\ Dialysis Center, NephroCare Ltd., \\ Fresenius Medical Care, Taichung, Taiwan; \\ ${ }^{6}$ Graduate Institute of Clinical Medical \\ Science, China Medical University, \\ Taichung, Taiwan \\ *These authors contributed equally to \\ this work
}

Correspondence: Chun-Ju Lin; Chun-Ting Lai

Department of Ophthalmology, China Medical University Hospital, 2 Yuh-Der

Road, Taichung City, 40447, Taiwan

Tel +886-4-22052121, ext. 1141

Fax +886-4-22059265

Email doctoraga@gmail.com;

withwind037@yahoo.com.tw
This article was published in the following Dove Press journal: Clinical Ophthalmology

Background: To evaluate the efficacy of micro-incision vitrectomy surgery using a noncontact wide-angle viewing system for fovea-attached type epiretinal membrane, and to report the factors influencing the outcome.

Methods: A retrospective, comparative case series that included 50 patients with foveaattached type epiretinal membrane who received micro-incision vitrectomy surgery using a non-contact wide-angle viewing system.

Results: All patients were followed-up for a minimum of 12 months. Seven cases were classified as group 1A (mainly outer retinal thickening), 17 were group 1B (more tenting of outer retina and distorted inner retina), and 26 were group $1 \mathrm{C}$ (prominent inner retina thickening and inward tenting of outer retina). Outcome measures included operation time, recurrent rate, postoperative BCVA, and CRT. The mean operative time was 26.2 minutes. The mean change of BCVA ( $\operatorname{LogMAR})$ was $-0.43(\mathrm{p}<0.001)$. The mean change of CRT was $135.3 \mu \mathrm{m}(\mathrm{p}<0.001)$. The mean change of CRT was significantly higher in group 1C. Worse preoperative BCVA, male gender, and longer operative time can predict better postoperative BCVA found by multivariate logistic regression and multiple regression models.

Conclusion: Significant improvement in BCVA and CRT is noted after micro-incision vitrectomy surgery to operate fovea-attached type epiretinal membranes. Worse preoperative BCVA, male, and longer operation time could predict better improvement. These findings may assist surgeons in better evaluating the potential of this method to help their patients with epiretinal membranes.

Keywords: fovea-attached type epiretinal membrane, gender, micro-incision vitrectomy surgery, operation time, preoperative vision, wide-angle viewing system

\section{Background}

Epiretinal membranes (ERM) are avascular fibrocellular sheets that form on the inner retinal surface. Even though early stages are usually asymptomatic, with further progression, ERM distorts and induces tractional changes on the macula leading to visual impairment. ${ }^{1-3}$ Fovea-attached type ERM can be classified as Group 1A (ERM with mainly outer retinal thickening and maintained a nearly normal configuration), Group 1B (more exaggerated tenting of outer retinal layer in the fovea area and inner retina distorted by centripetal and anteroposterior forces due to ERM), and Group 1C (prominent inner retina thickening with inward tenting of the outer retinal reflectivity in the foveal area). ${ }^{4}$ Group $1 \mathrm{~A}$ and $1 \mathrm{C}$ are globally adherent to the retina, while Group $1 \mathrm{~B}$ is focally adherent (Figure 1). 


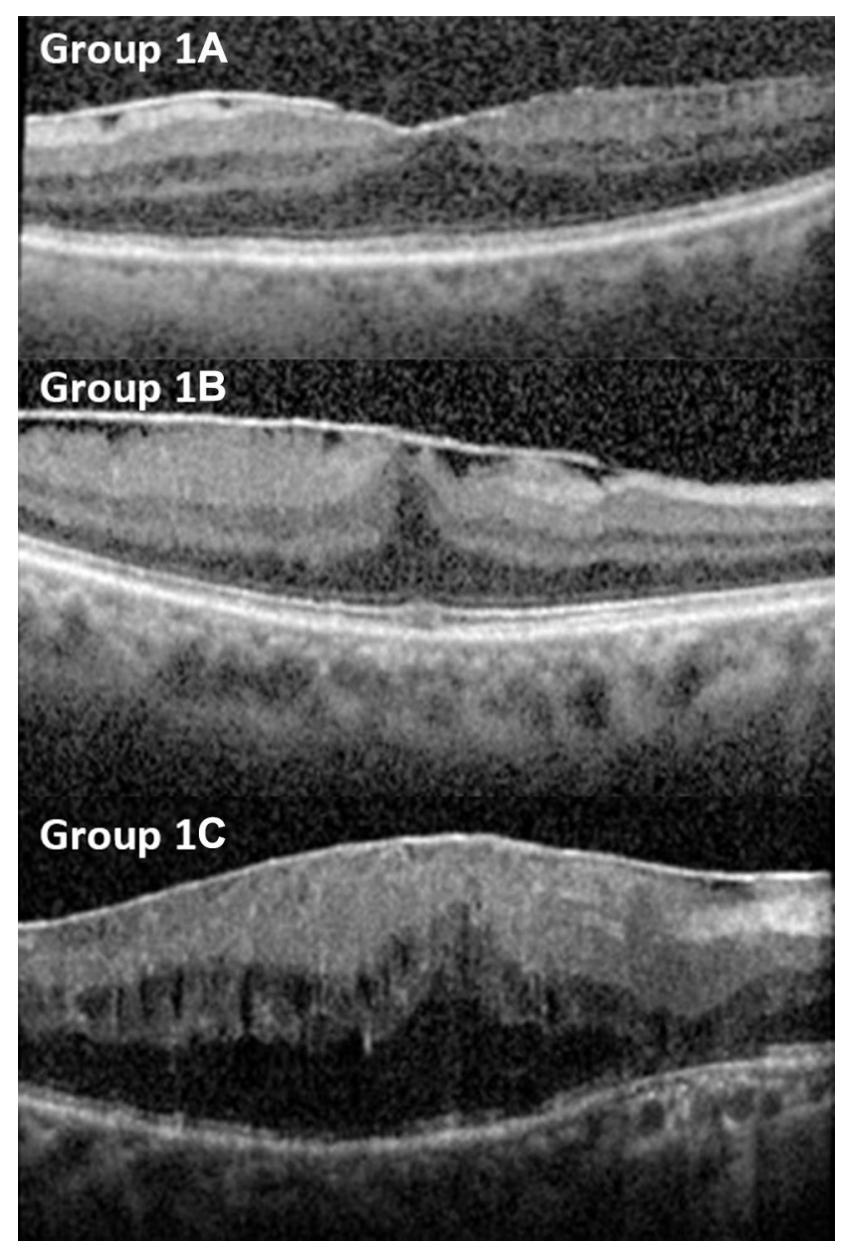

Figure I Fovea-attached type ERM can be classified as Group IA: ERM with mainly outer retinal thickening and maintained a nearly normal configuration, Group IB: more exaggerated tenting of outer retinal layer in the fovea area and inner retina distorted by centripetal and anteroposterior forces due to ERM, and group IC: prominent inner retina thickening with inward tenting of the outer retinal reflectivity in the foveal area. Group IA and IC are globally adherent to the retina, while Group IB is focally adherent.

There are two main management strategies: observation for spontaneous separation or surgical peeling. However, there is no consensus on the appropriate timing of intervention. ${ }^{5}$ Due to technical challenges in the past, pars plana vitrectomy (PPV) was usually reserved for a limited group of patients with severely affected visual functions.

Recently, with the advances in vitreoretinal surgery, safer and more effective surgeries are now performed. ${ }^{6-10}$ Microincision vitrectomy surgery (MIVS) reduces sutures' discomfort and postoperative inflammation. Moreover, the operative time is significantly decreased, which lessens perioperative patients' discomfort. Generally, indication for surgery includes patient-reported metamorphopsia, diplopia, or vision reduction to less than 20/60.

Innovations to the viewing system include the contact lens and noncontact lens wide-angle viewing systems
(WAVS). The RESIGHT (Carl Zeiss Meditec) WAVS contains two fixated lenses (128D and 60D) that could be rotated easily. Noncontact sutureless WAVS, unlike contact WAVS, requires neither sutures nor the aid of skilled assistants to hold the lens in place during surgery. ${ }^{11,12}$

Prognostic factors that have been reported include bestcorrected visual acuity (BCVA), central retinal thickness (CRT), pseudohole, cystoid macula edema, intact photoreceptor inner and outer segments, location of ERM, and ocular perfusion. ${ }^{3,5,13}$ In this study, we report the functional and anatomical outcomes of the MIVS using WAVS in fovea-attached type ERM. In addition, we discuss the prognostic factors which contribute to better visual improvement.

\section{Methods}

The study protocol was conducted according to the principles described in the Declaration of Helsinki. This study was approved by the Institutional Review Board of China Medical University Hospital, Taiwan. A retrospective review was performed on all medical records coded between June, 2015 and November, 2017 with the operation code microincision vitrectomy surgery and ICD-9 diagnosis code 362.56 (macular puckering) yielding 160 cases. Cases were excluded: 1) if there was less than 1 year of follow-up data; 2) if there was a history of other retina diseases including macular hole, proliferative diabetic retinopathy, retinal detachment, diabetic macular edema, age-related macular degeneration, choroid neovascularization or uveitis; 3) previous vitrectomy in the eye.

Further classification was done by reviewing spectraldomain optical coherence tomography (OCT, Heidelberg Engineering, Heidelberg, Germany) reports according to the consensus of three ophthalmologist (WH Kung, CT Lai, CJ Lin). Cases with type 2 ERM (pseudohole type) were also excluded. ${ }^{4}$ This yielded 50 eyes of 50 patients that were included in the study. These eyes with idiopathic ERM were treated with MIVS using WAVS from June 2015 till November 2017 by a single surgeon (C.J. L.). Preoperatively, patient demographics, refraction status, and BCVA were measured along with OCT, intraocular pressure (IOP), lens status, and comorbidities. The BCVA were measured using a projected Snellen chart. The same visual acuity method was used to measure BCVA preoperatively and postoperatively. Data were also recorded postoperatively during follow up for at least 1 year. Outcomes measurement was focused on operation time, BCVA, and CRT changes. 


\section{Surgical Procedure}

The indications of surgical intervention are 1) Reduction in visual acuity to worse than $20 / 60$ and 2) Moderate reduction in visual acuity but with annoying metamorphopsia or occupational concerns. All surgeries were performed after administration of retrobulbar anesthesia. Trocar and infusion cannula were inserted. 25-gauge threeport MIVS was performed using the Alcon Constellation vitrectomy system (Ft Worth, TX, USA) and Lumera 700 and Resight (Carl Zeiss Meditec) noncontact type WAVS.

Core vitrectomy was performed with careful attention under 128D lens. Intravitreal triamcinolone was used in all cases to identify and remove the vitreous and epiretinal membranes as completely as possible. Additional "heavy indocyanine green (ICG)" (mix ICG powder $25 \mathrm{mg}$ with

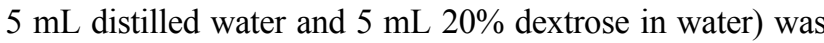
then injected intravitreally for internal limiting membrane (ILM) staining. ICG sits on the macula for about 10 seconds and is removed. And then the membranes were peeled by end grasping forceps (Alcon Grieshaber-Switzerland/Alcon Labs, Inc., Fort Worth, TX, USA) in either outside-in technique or inside-out technique under a 60D lens.

Scleral depression was performed under a 128D lens to check for possible iatrogenic breaks. Fluid-air exchange with a soft-tip extrusion needle and active suction to drain posterior pole fluid was done in selected cases, such as preexisting or iatrogenic breaks. At the conclusion of each procedure, levofloxacin eyedrops were placed on the surface of the eye followed by a patch and shield.

\section{Statistical Analysis}

Statistical analyses were performed using SPSS for Windows (Version 23.0, Statistical Package for the Social Sciences; SPSS, Inc, Chicago, IL). P value 0.05 was considered statistically significant. Numerical results were expressed as the mean \pm standard deviation (SD). For calculation of the mean and subsequent statistical comparison, BCVA was converted to logarithm of the minimum angle of resolution units (logMAR) for analysis. Descriptive statistics, including mean and SD, were calculated for patient characteristics. Two-tailed Student's $t$-test was used to compare means, and a chi-square test was performed for comparisons of categorical variables. ANOVA and Pearson correlation coefficient were used to analyze associations of different subtypes between preoperative BCVA, preoperative CRT, postoperative BCVA and postoperative CRT.
Because it will be quite meaningful to detect possible clinical factors that will influence the surgical outcome, multivariate logistic regression and multiple regression were used to build models for predicting postoperative visual outcome. The difference between these two methods is that categorical variable used as the dependent variable in logistic regression (in our case, the dependent variable is "satisfactory surgical result or not", which is defined by $\triangle B C V A$ LogMAR $<-0.4$ ) and the continuous variable used as the dependent variable in multiple regression (in our case, it is the extend of BCVA improvement after treatment, which is defined by $\triangle \mathrm{BCVA}$ LogMAR (postoperative BCVA minus preoperative BCVA)).

In the initial model of both methods, most of the possible influencing clinical factors were included as follows: 1) gender 2) age 3) diabetes 4) diagnosis 5) ERM classification 6) operation time in minutes 7) preoperative visual acuity 8) preoperative IOP 9) preoperative OCT 10) cataract surgery (phakic or pseudophakic) 11) postoperative IOP change. All these factors were used as initial independent variables and then the model selection was performed with stepwise selection method with entry and stay significant level set at 0.05 . Only factors passing these sieving procedures remained in the final models. Using two methods with different statistical principles to build prediction models, we wish we can identify more possible predicting factors.

\section{Results}

Of the 50 eyes that met the inclusion criteria, the mean age was $68.6(61.0-76.1)$ years old. Eighteen eyes (36\%) were male and 32 eyes (64\%) were female. The lens statuses were $80 \%$ phakic and $20 \%$ pseudophakic. Twenty-two percent of patients had diabetes. The ERMs were classified according to OCT appearance. Seven eyes (14\%) were labeled as type $1 \mathrm{~A} ; 17$ eyes (34\%) were labeled as type $1 \mathrm{~B} ; 26$ eyes $(52 \%)$ were labeled as type1C.

The mean preoperative LogMAR BCVA was 0.65 (range 0.37-0.94), mean preoperative CRT was 500.6 um (range 408.3-592.9), mean preoperative IOP was 15.5 (range 12.5 18.6) $\mathrm{mmHg}$, and mean operation time was 26.2 minutes (range 20.5 31.9). Twenty percent of patients received fluid-air exchange at the end of the procedure for preexisting or iatrogenic retinal breaks. No iatrogenic lens trauma at the trocar entrance was noted intraoperatively.

Postoperatively, the mean LogMAR BCVA was 0.22 (range -0.07-0.51); the mean CRT was 365.3 um (range 313.0 417.6); the mean IOP was $15.1 \mathrm{mmHg}$ (range 
12.5-17.6). The most common complication was IOP elevation which anti-glaucoma medication was necessary, occurring in $9(18 \%)$ patients. Furthermore, 34 out of the 40 phakic eyes $(85 \%)$ received cataract surgery within the follow-up period (Table 1).

Outcome analysis demonstrates significant BCVA and CRT improvement after MIVS. The mean change of BCVA in LogMAR is $-0.43(p<0.001)$. The mean change of CRT is -135.3 um $(p<0.001)$. Comparing subgroups of ERM Type 1A, 1B, 1C, ANOVA showed significant differences in change of CRT. However, there was no difference in operation time or BCVA improvement among subgroups (Table 2). In order to eliminate the influence of cataract, a subanalysis was done to analyze the BCVA outcome of the pseudophakic group. Eyes were grouped by those who were pseudophakic from the start (preoperative pseudophakic eyes) and those initially phakic with subsequent cataract surgery (preoperative phakic eyes with subsequent cataract surgery). Pearson correlation coefficient analysis showed a -0.43 correlation of BCVA improvement with operation time; and a +0.43 correlation of BCVA improvement with patient's age.

We also searched for predictive factors of the outcome of ERM removal using sutureless MIVS with non-contact WAVS. We defined satisfactory surgical result as $\triangle \mathrm{BCVA}$ $\operatorname{LogMAR}<-0.4$, which is the median value of our data. Possible influencing factors such as sex, age, right or left eye, diabetes, ERM classification, operation time, preoperative BCVA, preoperative IOP, preoperative CRT, history of cataract surgery, postoperative IOP elevation, and air-fluid exchange were included in the analysis. Using $\triangle \mathrm{BCVA}$ LogMAR $<-0.4$ or $>-0.4$ as the dependent variable, multivariate logistic regression analysis demonstrated that male gender and worse preoperative BCVA were two factors that contributed to better BCVA improvement (Table 3). The area

Table I Patient Postoperative Demographics

\begin{tabular}{|l|r|}
\hline Total Number of Eyes & 50 Eyes \\
\hline Mean Postoperative IOP $(\mathrm{mmHg})$ & I5.I (I2.5-I7.6) \\
\hline Mean Postoperative BCVA (LogMAR) & $0.22(-0.07-0.5 \mathrm{I})$ \\
\hline Mean Postoperative CRT $(\mu \mathrm{m})$ & $365.3(3 \mathrm{I} 3.0-4 \mathrm{I} 7.6)$ \\
\hline Cataract surgery, N & $34(85 \%)$ \\
\hline Complications, N & $9(\mathrm{I} \%)$ \\
Elevated IOP (medication necessary) & 0 \\
ERM Recurrence & 0 \\
Endophthalmitis & \\
\hline
\end{tabular}

Table 2 ANOVA ERM Subgroup Analysis

\begin{tabular}{|l|r|r|r|}
\hline $\begin{array}{l}\text { ERM } \\
\text { Classification }\end{array}$ & $\begin{array}{r}\text { Operation } \\
\text { Time }\end{array}$ & $\Delta$ BCVA & $\Delta$ CRT \\
\hline IA (7) & 25.3 & -0.50 & -57.0 \\
& $(21.0 \sim 29.6)$ & $(-0.74 \sim-0.26)$ & $(-93.1 \sim-20.9)$ \\
\hline IB (17) & 25.8 & -0.43 & -132.8 \\
& $(18.9 \sim 32.3)$ & $(-0.59 \sim-0.27)$ & $(-198.8 \sim-66.8)$ \\
\hline IC (26) & 26.7 & -0.42 & -158.0 \\
& $(21.6 \sim 31.8)$ & $(-0.68 \sim-0.16)$ & $(-229.0 \sim-87.0)$ \\
\hline ANOVA & $\mathrm{P}=0.8069$ & $\mathrm{P}=0.7178$ & $\mathrm{P}=0.004 \mathrm{I}$ \\
\hline
\end{tabular}

Abbreviations: BCVA, best-corrected visual acuity; ERM, epiretinal membrane; CRT, central retinal thickness.

under curve of the model was 0.767 with acceptable discrimination.

These findings were supported by the significantly higher male proportion (Figure 2A) and significantly worse mean preoperative BCVA (Figure 2B) in the group with better visual improvement. Using $\triangle \mathrm{BCVA} \operatorname{LogMAR}$ as the continuous dependent variable, multiple regression analysis showed that longer operation time and worse preoperative BCVA were factors that were significantly related to better visual improvement (Table 3). These findings were supported by the significant correlation between these two parameters (operation time, preoperative BCVA) and the degree of improvement of BCVA after MIVS (The Pearson correlation coefficient were -0.429 and $-0.387, \mathrm{p}<0.05$ )

Indeed, we included phakic/pseudophakic status as one of the possible influencing factors in the initial models of logistic and multiple regression. However, this factor of phakic/pseudophakic status was not included in the final models from both analyses, which meant cataract surgery itself was not as influencing as the final factors remaining in the models for the visual outcome from the results of multivariate analyses (Table 3).

\section{Discussion}

Idiopathic ERMs are fibrocellular membranes on the vitreoretinal interface. While it may be asymptomatic or separate spontaneously, metamorphopsia and impaired central vision are major concerns with its progression. ${ }^{1}$ Sutureless MIVS and WAVS have been widely applied for ERM management. ${ }^{4,6,9,12}$ In this case series, 50 eyes of 50 patients with idiopathic ERM underwent 25-gauge MIVS with ERM and ICG-assisted ILM peeling using non-contact WAVS and followed up for at least 12 months. All MIVS were under retrobulbar anesthesia and performed by the same surgeon to lessen bias. 
Table 3 Multivariate Models for Predicting Operation Outcome

\begin{tabular}{|l|l|l|l|l|l|}
\hline \multicolumn{2}{|l|}{ Multivariate Logistic Regression } \\
\hline Parameters & Estimate & Standard Error & Wald Chi-Square & Odds Ratio & P value \\
\hline Intercept & -1.78 & 0.90 & 3.92 & & 0.22 \\
If Male & 0.72 & 0.33 & 4.75 & 13.82 & $0.04^{*}$ \\
BCVA $_{\text {pre }}$ & 2.63 & 1.29 & 4.17 & & $0.02^{*}$ \\
\hline Multiple Linear Regression & \multicolumn{5}{|l|}{ F Value } \\
\hline Parameters & Estimate & Standard Error & Type II SS & 0.24 & P value \\
\hline Intercept & 0.07 & 0.14 & 0.01 & 6.19 & 0.62 \\
Operation Time $_{\text {BCVA }}$ & -0.01 & 0.01 & 0.26 & 4.10 & $0.01^{*}$ \\
\hline
\end{tabular}

Note: ${ }^{*} p<0.05$.

Abbreviation: BCVApre, preoperative BCVA (logMAR).

Our study showed an improvement in the mean BCVA from 0.65 to 0.22 LogMAR after 12 months. These results are in line with Dawson et al, who reported the outcomes of standard PPV in 237 patients over 10 years showing a similar improvement of BCVA from 0.6 to 0.3 LogMAR on a median follow-up of 0.55 years. ${ }^{13}$ For sutureless vitrectomy, Reibaldi et al conducted a randomized control trial to compare 25- and 27-gauge systems in ERM management. ${ }^{8}$ The study enrolled 41 phakic patients in the 25-gauge ERM group showing a significant improvement in BCVA from 0.41 to $0.27 \operatorname{LogMAR}$ after 1 year. This discrepancy in visual gain may be partially attributed to the non-use of dye in ERM peeling which may have affected the competency in the peeling process. Another study by Rizzo et al reported a significant improvement of median BCVA from 20/150 to 20/83.5 at 6 months after 25-gauge PPV. ${ }^{14}$

Aiming to emphasize concurrent results, prior studies have compared the 25- and 27-gauge systems. Naruse et al recently reported the outcomes of both systems performed in 200 ERM patients; 100 in each group. ${ }^{4}$ The 27 -gauge system achieved better visual functions at 1 month, while no significant differences were encountered in the visual gain for either groups at 6 months (7.8 and 6.4 in 27- and 25-gauge systems, respectively). In addition, many studies have compared the 20- vs 25 gauge systems, ${ }^{14,15}$ and the 23 - vs 25 -gauge systems. ${ }^{16}$ These studies showed that smaller gauge systems (25-gauge) achieved more rapid visual recovery supported by better outcomes at 1 month, but comparable outcomes at longer followups.

Besides improvement in visual acuity, CRT is considered another indicator for the effectiveness of the procedure applied. In our series, 25-gauge MIVS significantly lowered the CRT from $500.6 \mu \mathrm{m}$ to $365.3 \mu \mathrm{m}$. Reibaldi et al reported similar outcomes with 25 -gauge vitrectomy, achieving CRT of $275 \mu \mathrm{m}$ at 12 months compared to an average of $457 \mu \mathrm{m}$ preoperatively. ${ }^{6}$ However, this change was not significantly different from the 27 -gauge system (289 $\mu \mathrm{m}$ at 12 months from $452 \mu \mathrm{m}$ preoperatively). In agreement with Reibaldi, Naruse et al found no significant difference in CRT reduction between the 25- and 27-gauge systems at 3 and 6 months after surgery. ${ }^{4}$

Operation time is another important factor for the functional outcomes in ERM. This factor impacts patients' comfort and postoperative inflammation. In our series, the mean operation time was 26.2 minutes which is closely similar to the mean operation time of 25 -gauge (32.7 minutes) and 27 -gauge (36.7 minutes) reported by Naruse. ${ }^{4}$ However, it is a bit different from Rizzo et al who reported a mean of 15.6 minutes in the 25 -gauge and 29.6 minutes in the 20-gauge group. ${ }^{14}$ Therefore, it is evident that operation time can be influenced by many factors rather than the system's gauge size. This includes clinical condition, surgeon's experiences, and other technical supplies.

The safety of surgical procedures is another concern. Although complications are rare, cataract progression and IOP changes are the most common complications with a 25 -gauge vitrectomy. ${ }^{17}$ In our series, $85 \%$ of our patients underwent cataract surgery after the ERM surgery during the 12-month follow-up. This is in accordance with many studies with lens-sparing vitrectomy. ${ }^{17,18}$ It is well known that vitrectomy surgery causes the progression of nuclear cataract. In our cases, intraocular triamcinolone was removed as completely as possible and no iatrogenic lens 

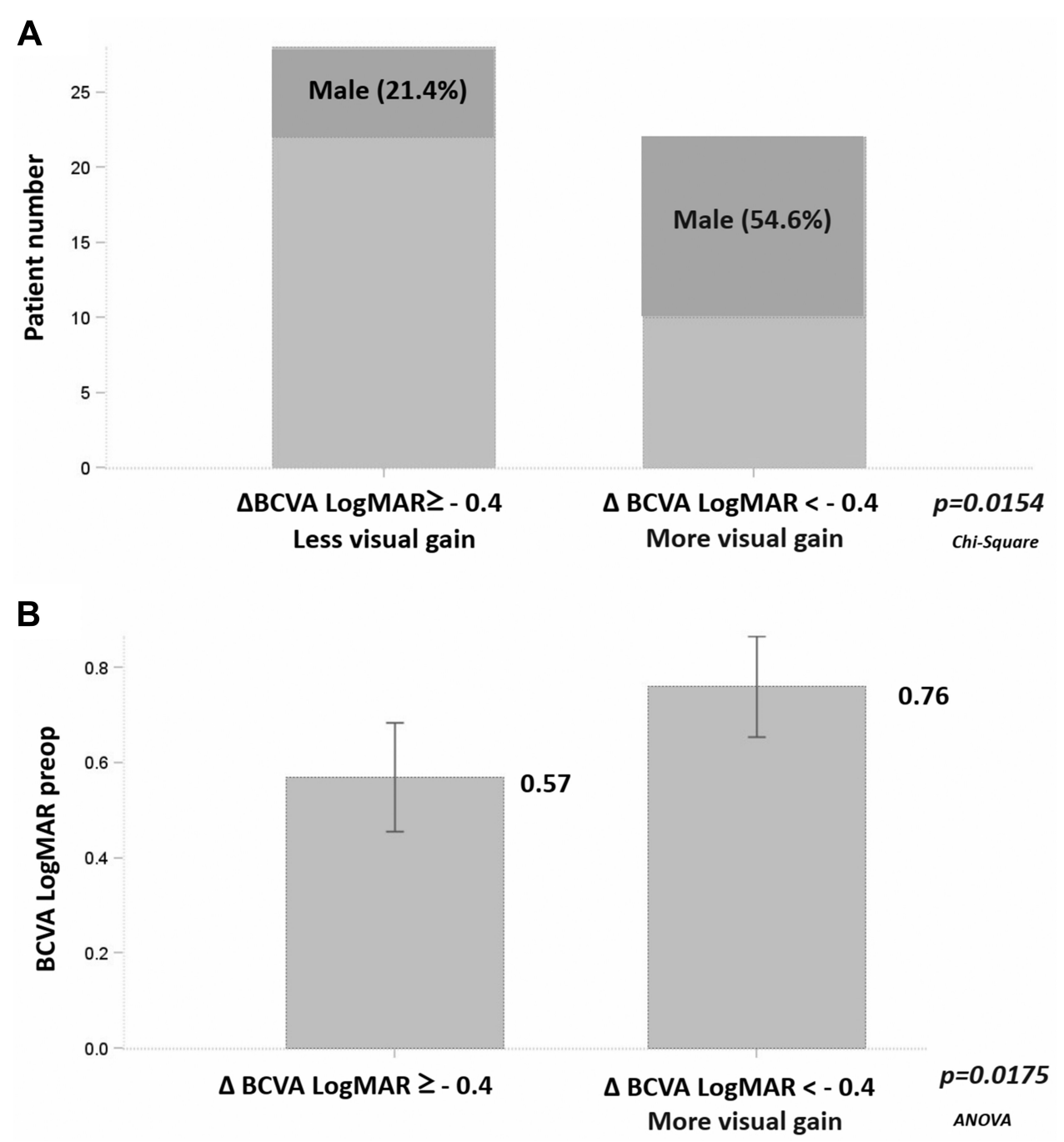

Figure 2 (A) Comparison of proportion of male gender between groups with different visual outcomes. (B) Comparison of preoperative BCVA (LogMAR) between groups with different visual outcomes.

trauma at the trocar entrance was noted intraoperatively. One possible mechanism is that molecular oxygen from the retina reaches the lens and induces oxidative damage to the lens nucleus. Some authors recommend combined cataract surgery and vitrectomy for idiopathic ERM with promising outcomes. ${ }^{12}$

Elevated IOP is among the most frequently reported complications. In a sutureless procedure, hypotony is a concern as well as IOP elevation due to postoperative inflammation. In our series, IOP elevation was encountered in nine $(18 \%)$ patients with no hypotony reported. In the literature, elevated IOP was reported in multiple studies with variable rates ranging from $0 \%(5)$ to $4.5 \% .{ }^{17}$ In addition, hypotony was also reported. ${ }^{6,17,18}$ The rate of hypotony or IOP elevation was lower with the 27 -gauge system compared to the 25 -gauge system. This supports the theory that smaller gauge systems have a stable IOP course.
Postoperative transient hypotony is caused by transient leakage and ciliochoroidal detachment but usually resolves spontaneously by 1 week after the operation. ${ }^{17,19-21}$

Prognostic factors for visual gain after sutureless MIVS are variable. However, preoperative low visual acuity and the male gender were the two most important factors in our series, as determined by logistic regression. Among the major studies for prognostic factors of visual gain, Song et al reported the outcomes of 504 eyes with idiopathic ERM that underwent PPV. ${ }^{3}$ Controlling age, duration of symptoms, and CRT, poor preoperative BCVA along with preoperative pseudophakia were the only significant prognostic factors for a visual gain of at least 2 Snellen lines. A preoperative BCVA of $0.51 \operatorname{LogMAR}$ was the optimal cutoff value for a visual gain of at least 2 Snellen lines at 3 months. Moreover, the AUC was 0.66 , which approaches the AUC result of our series (0.767). 
To the best of our knowledge, the finding of the male gender as a predictive factor for better postoperative outcomes has not previously been published. Previous studies on the outcomes of epiretinal membrane surgery have not reported outcome differences based on gender. ${ }^{16,18,19,22,23}$ Given the statistical significance of the association between male gender and better postoperative BCVA in this study, it is less likely to be the result of statistical bias. One tentative explanation for the association is the possible innately better visual function of men: prior research investigating gender differences in visual perception has found that males performed significantly better than females in several key aspects of vision, including visual acuity and motion detection. However, no patterns suggestive of specific neurological, cognitive, or hormonal explanations for the differences were determined. ${ }^{24,25}$ We also considered the contribution of hormonal effects on inflammation and postoperative tissue recovery. However, studies have largely found androgens to be pro-inflammatory and estrogen to mediate the resolution of inflammation and tissue repair. ${ }^{26,27}$ Prospective studies with larger samples sizes focusing on the effects of gender are needed to support and further quantify our finding that males experience better outcomes after MIVS for epiretinal membrane. The finding from multiple regression that longer operation time predicted better visual improvement was also interesting. If the rationale behind this finding was similar to the one related to preoperative BCVA, cases in a more complicated situation might even benefit more from this procedure and deserve earlier aggressive management.

In contrast to our series, there are some studies that showed less visual gain after 25 -gauge vitrectomy $(0.13$ LogMAR at 6 months compared to 0.24 LogMAR at baseline). ${ }^{9}$ These studies selected patients with good baseline visual acuity (20/40 at least). This may explain these negative results in a visual gain. Besides low visual gain, these studies also showed a lower reduction in CRT. ${ }^{13}$ Nakashizuka et al reported nonsignificant change in CRT after 25-gauge vitrectomy for ERM, either with or without pseudo-macular hole (318.9 $\mu \mathrm{m}$ from $337 \mu \mathrm{m}){ }^{5}$ In our series, only fovea-attached type ERM were included and pseudohole type ERM was excluded, which lessened this selection bias.

There are several limitations to this study. This study was retrospective and the sample size was relatively small with the lack of comparison with other sutureless systems. Assessment of the metamorphopsia quantification is also another limitation to be considered in future work. Nevertheless, the comparison between preoperative and intraoperative variables was made and showed significant results.

\section{Conclusions}

In summary, sutureless MIVS using non-contact WAVS is a safe and effective procedure for fovea-attached type ERM management. While recent several reports encourage surgery for those with relatively good vision, our study demonstrated that patients with worse preoperative BCVA, male gender, and relatively longer operation time are more likely to gain better visual outcomes. Prospective and randomized studies would help further corroborate these findings.

\section{Abbreviations}

BCVA, best-corrected visual acuity; CRT, central retinal thickness; ERM, epiretinal membrane; ICG, indocyanine green; ILM, internal limiting membrane; IOP, intraocular pressure; MIVS, micro-incision vitrectomy; OCT, optical coherence tomography; PPV, pars plana vitrectomy; WAVS, wide-angle viewing system.

\section{Data Sharing Statement}

The datasets used and/or analysed during the current study are available from the corresponding author on reasonable request.

\section{Ethics Approval and Consent to Participate}

The study protocol described here was approved by the Institutional Review Board of China Medical University Hospital and conformed to the tenets of the Declaration of Helsinki.

\section{Consent for Publication}

All patients have provided written informed consent for the publication of their data and images.

\section{Funding}

No funding was used for the design and conduction of this study.

\section{Disclosure}

The authors have no proprietary, commercial, or noncommercial interest in any materials mentioned in this article. The authors report no conflicts of interest in this work. 


\section{References}

1. Iwanoff A. Beiträge zur normalen und pathologischen Anatomie des Auges. Graefes Arch Clin Exp Ophthalmol. 1865;11:135-170. doi:10.1007/BF02720906

2. Bu S-C, Kuijer R, Li X-R, Hooymans JMM, LI L. Idiopathic epiretinal membrane. Retina. 2014;34(12):2317-2335. doi:10.1097/ IAE.0000000000000349

3. Miguel AIM, Legris A. Prognostic factors of epiretinal membranes: a systematic review. $J$ Fr Ophthalmol. 2017;40(1):61-79. doi:10.1016/j.jfo.2016.12.001

4. Hwang JU, Sohn J, Moon BG, et al. Assessment of macular function for idiopathic epiretinal membranes classified by spectral-domain optical coherence tomography. Invest Ophthalmol Vis Sci. 2012;53 (7):3562-3569. doi:10.1167/iovs.12-9762

5. Song SJ, Kuriyan AE, Smiddy WE. Results and prognostic factors for visual improvement after pars plana vitrectomy for idiopathic epiretinal membrane. Retina. 2015;35(5):866-872. doi:10.1097/ IAE.0000000000000406

6. Naruse S, Shimada H, Mori R. 27-gauge and 25-gauge vitrectomy day surgery for idiopathic epiretinal membrane. $B M C$ Ophthalmol. 2017;17(1):1-7. doi:10.1186/s12886-017-0585-1

7. Nakashizuka H. Short-term surgical outcomes of 25 -gauge vitrectomy for epiretinal membrane with good visual acuity. J Clin Exp Ophthalmol. 2013;4(3). doi:10.4172/2155-9570.1000280

8. Reibaldi M, Longo A, Avitabile T, et al. Transconjunctival nonvitrectomizing vitreous surgery versus 25 -gauge vitrectomy in patients with epiretinal membrane: a prospective randomized study. Retina. 2015;35(5):873-879. doi:10.1097/IAE.0000000000000459

9. Fujii GY, De Juan E, Humayun MS, et al. A new 25-gauge instrument system for transconjunctival sutureless vitrectomy surgery. Ophthalmology. 2002;109(10):1807-1812; discussion 1813. doi:10.1016/S0161-6420(02)01179-X

10. Sayed KM, Naito T, Farouk MM, et al. Twenty five-gauge sutureless vitrectomy versus 20 -gauge vitrectomy in epiretinal membrane surgery. J Med Investing. 2012;59(1,2):69-78. doi:10.2152/jmi.59.69

11. Inoue M. Wide-angle viewing system. Dev Ophthalmol. 2014;54:87-91.

12. De Oliveira PRC, Berger AR, Chow DR. Vitreoretinal instruments: vitrectomy cutters, endoillumination and wide-angle viewing systems. Int J Retina Vitreous. 2016;2:28-43. doi:10.1186/s40942016-0052-9

13. Rommel F, Brinkmann MP, Sochurek JAM, Prasuhn M, Grisanti S, Ranjbar M. Ocular blood flow changes impact visual acuity gain after surgical treatment for idiopathic epiretinal membrane. J Clin Med. 2020;9(6):1768. doi:10.3390/jcm9061768

14. Moisseiev E, Kinori M, Moroz I, Priel E, Moisseiev J. 25-gauge vitrectomy with epiretinal membrane and internal limiting membrane peeling in eyes with very good visual acuity. Curr Eye Res. 2016;41 (10):1387-1392. doi:10.3109/02713683.2015.1114654
15. Nakano Y, Nomoto H, Fukuda K, et al. Combined 25-gauge vitrectomy and cataract surgery with toric intraocular lens with idiopathic epiretinal membrane. J Cataract Refract Surg. 2013;39(5):686-693. doi:10.1016/j.jcrs.2012.11.029

16. Dawson SR, Shunmugam M, Williamson TH. Visual acuity outcomes following surgery for idiopathic epiretinal membrane: an analysis of data from 2001 to 2011. Eye. 2014;28(2):219-224. doi:10.1038/ eye.2013.253

17. Rizzo S, Genovesi-Ebert F, Murri S, et al. 25-gauge, sutureless vitrectomy and standard 20-gauge pars plana vitrectomy in idiopathic epiretinal membrane surgery: a comparative pilot study. Graefe's Arch Clin Exp Ophthalmol. 2006;244(4):472-479. doi:10.1007/ s00417-005-0173-6

18. Kadonosono K, Yamakawa T, Uchio E, Yanagi Y, Tamaki Y, Araie M. Comparison of visual function after epiretinal membrane removal by 20 -gauge and 25 -gauge vitrectomy. Am J Ophthalmol. 2006;142(3):513-515. doi:10.1016/j.ajo.2006.03.060

19. Kim M, Park YS, Lee DH, Koh HJ, Lee SC, Kim SS. Comparison of surgical outcome of 23-gauge and 25-gauge microincision vitrectomy surgery for management of idiopathic epiretinal membrane in pseudophakic eyes. Retina. 2015;35(10):2115-2120. doi:10.1097/ IAE. 0000000000000598

20. Gupta OPI, Weichel ED, Regillo CD, et al. Postoperative complications associated with 25 -gauge pars plana vitrectomy. Ophthalmic Surg Lasers Imaging. 2007;38(4):270-275. doi:10.3928/1542887720070701-01

21. Takashina H, Watanabe A, Tsuneoka H. Perioperative changes of the intraocular pressure during the treatment of epiretinal membrane by using 25- or 27-gauge sutureless vitrectomy without gas tamponade. Clin Ophthalmol. 2017;11:739-743. doi:10.2147/OPTH.S133775

22. Chuang LH, Wang NK, Chen YP, et al. Comparison of visual outcomes after epiretinal membrane surgery. Taiwan J Ophthal. 2012;2 (2):56-59. doi:10.1016/j.tjo.2012.03.002

23. Elhusseiny AM, Flynn HW, Smiddy WE. Long-term outcomes after idiopathic epiretinal membrane surgery. Clin Ophthalmol. 2020;14:995-1002. doi:10.2147/OPTH.S242681

24. Shaqiri A, Roinishvili M, Grzeczkowski L, et al. Sex-related differences in vision are heterogeneous. Sci Rep. 2018;8:7521. doi:10.1038/s41598-018-25298-8

25. Klein BEK, Klein R, Lee KE, Cruickshanks KJ. Associations of performance-based and self-reported measures of visual function. The Beaver Dam Eye Study. Ophthalmic Epidemiol. 1999;6:49-60. doi:10.1076/opep.6.1.49.1569

26. Villa A, Rizzi N, Vegeto E, Ciana P, Maggi A. Estrogen accelerates the resolution of inflammation. In macrophagic cells. Sci Rep. 2015;5:15224. doi:10.1038/srep15224

27. Straub RH. The complex role of estrogens in inflammation. Endocr Rev. 2007;28(5):521-574.
Clinical Ophthalmology

\section{Publish your work in this journal}

Clinical Ophthalmology is an international, peer-reviewed journal covering all subspecialties within ophthalmology. Key topics include: Optometry; Visual science; Pharmacology and drug therapy in eye diseases; Basic Sciences; Primary and Secondary eye care; Patient Safety and Quality of Care Improvements. This journal is indexed on PubMed
Central and CAS, and is the official journal of The Society of Clinical Ophthalmology (SCO). The manuscript management system is completely online and includes a very quick and fair peer-review system, which is all easy to use. Visit http://www.dovepress.com/ testimonials.php to read real quotes from published authors.

\section{Dovepress}

